\title{
Man-Machine-Environment Virtual Design and JACK Simulation of Rosa Roxburghii Picking Machine
}

\author{
Jianming Zhang ${ }^{1}$ and Bing luo ${ }^{1^{*}}$ \\ ${ }^{1}$ School of mechanical engineering, Guizhou University, Guiyang, Guizhou, China
}

\begin{abstract}
In order to improve the coordination between human and agricultural machinery, taking Rosa roxburghii picking machine as an example, a virtual design method based on human-machine-environment system, combined with three-dimensional digital simulation technology and human skeleton biology and other disciplines is proposed. Three-dimensional parametric modeling of Rosa roxburghii picking machine is carried out by using three-dimensional engineering software. In JACK simulation software, a digital human body model is created to analyse the comfort, accessibility and visibility of the operator. The coordination degree of agricultural machinery design in the human-machine-environment system can be judged in advance, and targeted optimization design can be carried out to achieve the purpose of improving the coordination between human and machinery in the human-machine-environment system and reduce the accident rate. This method shortens the research and development cycle of agricultural machinery and provides a reference for humanized design of agricultural machinery design.
\end{abstract}

\section{Introduction}

With the development of agricultural modernization, agricultural mechanization has brought high yield and high efficiency, but at the same time, the safety accidents caused by agricultural machinery have also brought adverse effects on the safety of farmers' lives and property. According to research, in 2012 alone, 5,461 agricultural machinery accidents occurred in China, 1989 people died and 4,284 people injured, caused in direct economic losses of more than RMB 40 million yuan [1]. From the point of view of agricultural machinery design, the two important factors causing the accident are: first, the layout design of agricultural machinery operation interface is unreasonable, and only the mechanical performance is emphasized in the design process of agricultural machinery products, ignoring the coordination of interaction between human and machinery; The second is the ignore the consideration of the environmental factors in the operation of agricultural machinery products. China's agricultural machinery's using environment has diversified characteristics, From hot Xinjiang to cold three northeast provinces, the environment is quite different. Once these two factors are not taken into account in the design process, to a certain extent, they will not only affect the work efficiency, but also affect the performance of agricultural machinery itself, and even pose a major threat to the safety of agricultural machinery operators. From another point of view, with the continuous progress of agricultural mechanization, people's expectations for the comfort and operating environment of agricultural machinery are also improving [2], so it is necessary to study the coordination of agricultural machinery under the human-machineenvironment system.

Virtual design and simulation technology integrates advanced knowledge of many disciplines. From design, assembly to production process, virtual simulation technology can be applied to carry out all-round dynamic simulation and evaluate the feasibility and reliability of products. Due to the reduction of the actual processing and inspection process, the cycle time of products development is reduced greatly and cost saving is achieved [3]. Virtual simulation technology has been widely used in the past ten years. Fei Yang and others proposed RULA method to classify tractor cab and evaluate and grade its handling comfort [4]. Jin Xiaoping et al proposed a man-machine interface layout reasoning method applied to the design and evaluation of the manmachine interface layout of the vehicle cab in the virtual environment [5]. WeiWei Wang et al carried out ergonomic simulation on the arrangement design of emergency rescue vehicles through JACK software, and optimized the design according to the simulation results [6]. Although scholars at home and abroad have done more research on the simulation of agricultural machinery design, assembly and operation, there is still a relative lack of simulation research on agricultural machinery under the condition of human-machineenvironment system.

In this study, taking Rosa roxburghii picking machine as an example, JACK man-machine simulation software was used to simulate, analyse and evaluate the humanmachine-environment and the comfort, accessibility and

"Corresponding author's e-mail: 1258132509@qq.com 
visibility in the operation process, thus guiding the optimal design.

\section{Virtual Design of Agricultural Machinery}

\subsection{Man-Machine-Environment System Engineering of Agricultural Machinery}

So far, the human-machine-environment system has gone through three stages of development: which is changing from machine-centered, application-centered to the concept "human-oriented"[7]. It consists of human (individuals and groups), machines (machines, mechanisms, functions and other parts), and environments (natural environment, social environment, task environment, cognitive environment and other fields) [8]. In the process of operation, the machine transmits information to people through auditory, tactile and olfactory senses. People process the information through the central nervous system and then direct moving organs such as hands and feet to operate the machine. Drivers will encounter various environmental factors (temperature, noise, vibration, harmful gases, etc.) during driving [9]. Fig 1 is the human-machine-environment system of agricultural machinery. Human represents the operator of agricultural machinery, machinery is agricultural machinery, and environment is the general environment in which agricultural machinery is located, such as natural and working environment such as roads and land, as well as social environment and user's cognitive level.

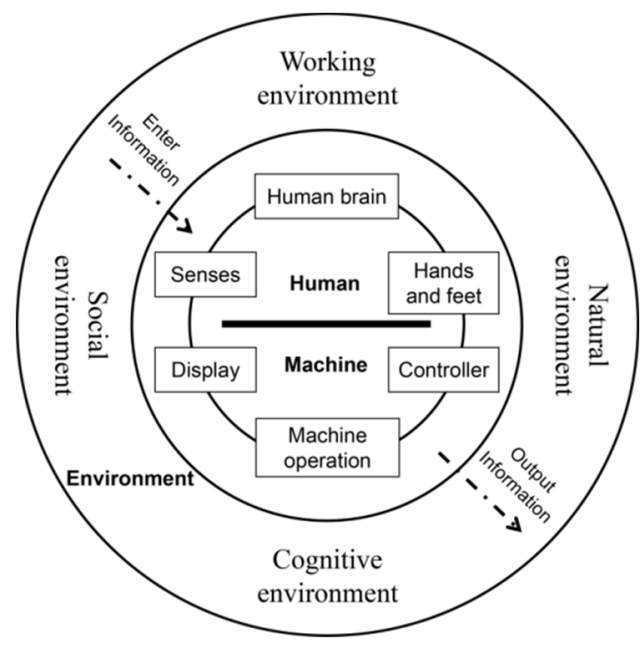

Fig 1. Schematic Diagram of Research Category of Agricultural Machinery Man-Machine-Environment System

\subsection{Virtual Design System of Agricultural Machinery under Man-Machine-Environment System}

The virtual design system of agricultural machinery under the human-machine-environment system is shown in Fig 2. Its purpose is to study the operation rationality and riding comfort of operators and agricultural machinery, continuously improve the parameters among various factors of people, machines and environment, and guide the optimal design. Among them, JACK human factor analysis includes comfort, accessibility and visibility analysis, such as human driving posture comfort, human operation range and spatial accessibility, human visibility in the operation process. The safety, high efficiency, reliability and other aspects of the agricultural machinery system are comprehensively evaluated, and the overall design scheme of the design object is finally determined.

From the perspective of analysing and solving the overall related problems of agricultural machinery, ProE, UG and other digital technologies are used to carry out parametric modeling on the appearance and structure of the provided parts. The constraint conditions of parts are strictly observed when we is modeling, and the complete parametric model of Rosa roxburghii picking machine is completed according to the principles of ergonomics. 


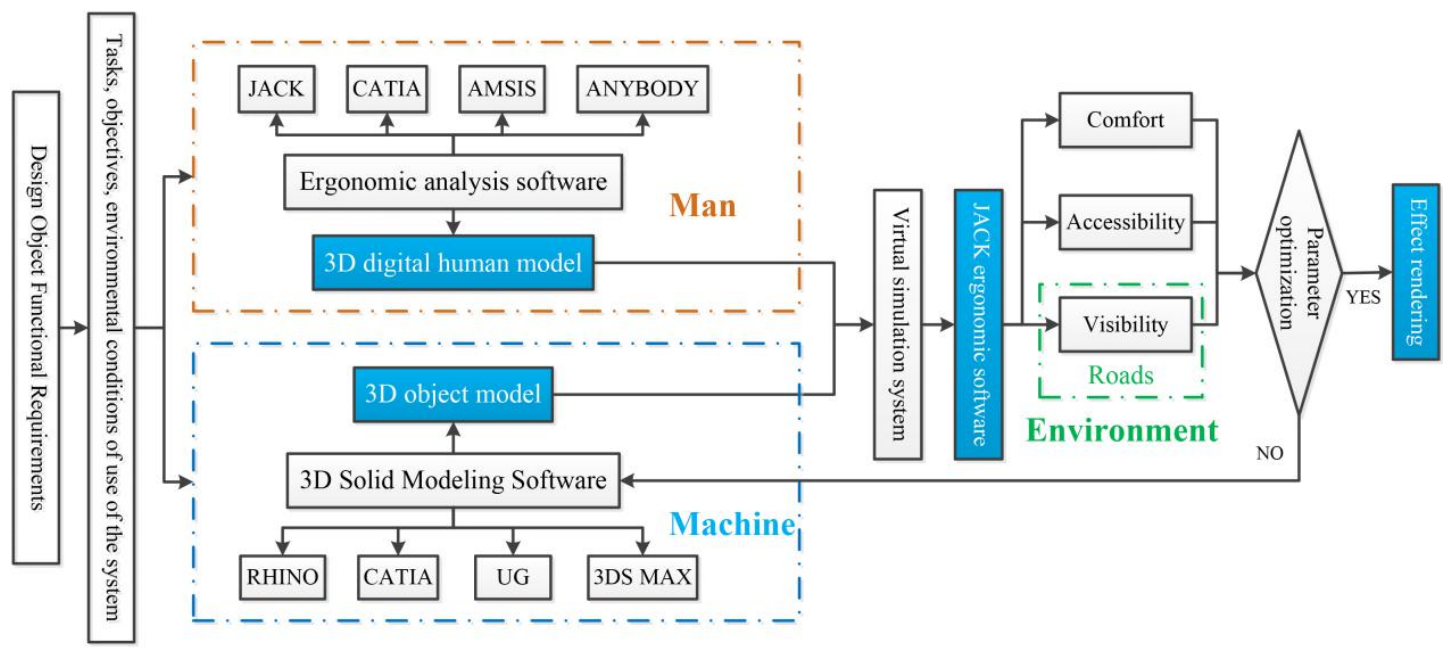

Fig 2. Virtual Design of Agricultural Machinery under Man-Machine-Environment System

\section{Case Application of Rosa roxburghii Picking Machine}

\subsection{Man-Machine-Environment System Engineering of Agricultural Machinery}

Taking Rosa roxburghii picking machine as an example, the virtual simulation system analysis is carried out. The first part is the construction of human body model. There are $5 \%, 50 \%$ and $95 \%$ human body size models in JACK database of human-machine efficacy system, which can comprehensively simulate the body size proportion of existing agricultural machinery operators. The second part is the construction of mechanical model and environmental model. This part is modelled and drawn by professional ProE and other three-dimensional software. Its purpose is to build agricultural machinery model one to one according to the needs of production functions, and to analyse the operation of mechanical parts in the simulation system to achieve the expected results. As shown in Fig 3 is JACK virtual simulation system of Rosa roxburghii picking machine.

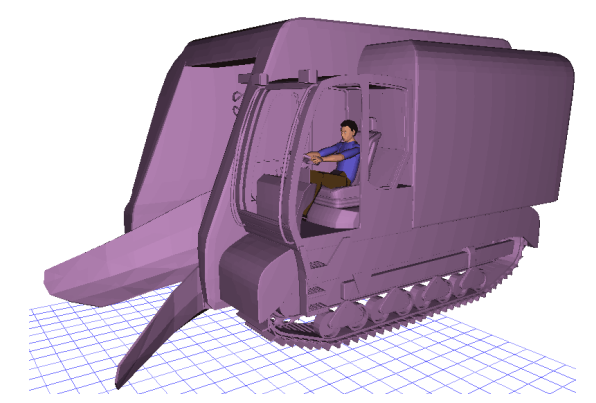

Fig 3. Shows JACK virtual simulation system of Rosa roxburghii picking machine

\subsection{Ergonomic Analysis of Rosa roxburghii Picking Machine}

The theory of modern human-machine-environment system is designed around human-centered analysis, of which the most basic characteristics of human are physiological characteristics. In the process of human operation, factors such as machines and environment need to reach the reasonable range of human physiological characteristic parameters [7]. Specifically, the machine design must meet the conditions of human physiological comfort, operability and visibility.

\subsubsection{Comfort analysis}

Good comfort can prevent the occurrence of occupational diseases to a certain extent and improve work efficiency [10]. JACK simulation system provides the comfort analysis of the joint parts of the human body in a certain action state, and can judge the comfort degree under the action. After constructing JACK manmachine simulation system, the evaluation index data of comfort degree are output. The evaluation index range of comfort degree of each part is between 0 and 80 , and the smaller the value, the better the comfort degree. As shown in Fig 4, for the analysis of the output data, Shoulder's data is 74.8, which indicates that the shoulder may not provide good support or the unreasonable distance between the shoulder and the operating lever causes poor comfort in this part during the operation. Neck's data is 61.7, and the comfort level is not good. The reason may be that the headrest angle or size provided does not support it enough. Fatigue's data reached 80 , indicating that the operator is extremely prone to physical fatigue during operation. Comfort has a value of 80 , indicating that the overall comfort level is extremely poor. Through comfort analysis, the comfort of various parts of the human body can be accurately fed back, thus giving accurate guidance to seat design, spatial layout, etc. According to the results of simulation feedback, the parameters of Rosa roxburghii picking machine, such as seat height, headrest size and angle, 
steering wheel angle and position, foot pedal height and angle, are optimized, and simulation analysis is carried

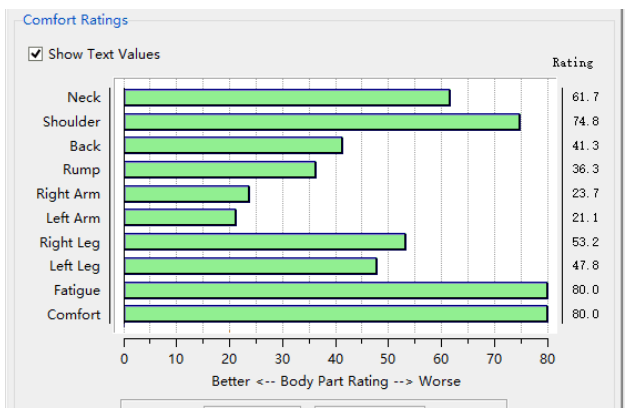

Fig 4. Analysis of digital human comfort

The drivers' behavior of agricultural machinery operation process is holding the steering wheel with one's hand and stepping on the operation pedal with one's foot. The action is simulated in JACK simulation system. Fig 6 is the static stress strength index output by the action. Knee and Ankle shows dangerous red warning, this means that the operator is extremely uncomfortable with his knees and ankles and is difficult to apply force

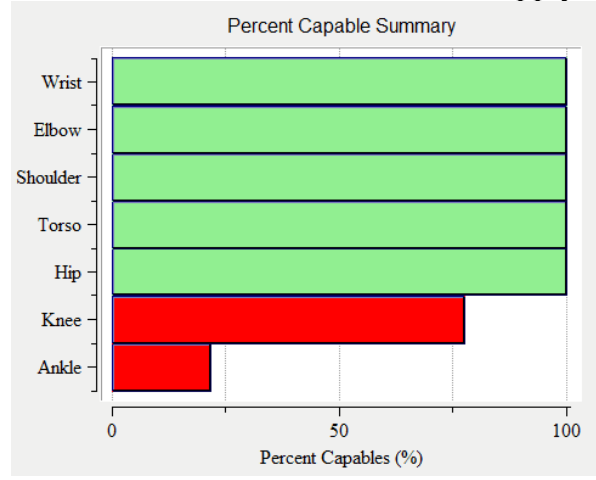

Fig 6. Static strength of driver

Research on human sitting posture shows that the position between L4 and L5 of lumbar spine is most prone to pathological changes when sitting posture is maintained for a long time. The reasons are unreasonable static pressure distribution in human sitting posture and high pressure on the position between L4 and L5 of lumbar spine in static sitting posture [11-12]. In a comfortable sitting position, the distribution of pressure

$$
\text { low back spinal forces ( } L 4 / L 5)
$$

out in JACK again. As shown in Fig 5, the comfort level has reached an ideal level.

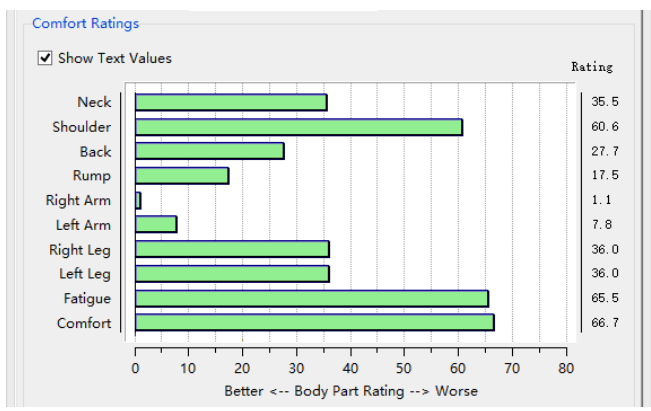

Fig 5. Analysis of digital human comfort after optimization

under this action. After many times of optimization, the simulation is carried out again. As shown in Fig 7, all static strength values have reached the green safety value, indicating that all static strength indexes have reached a relatively ideal level.

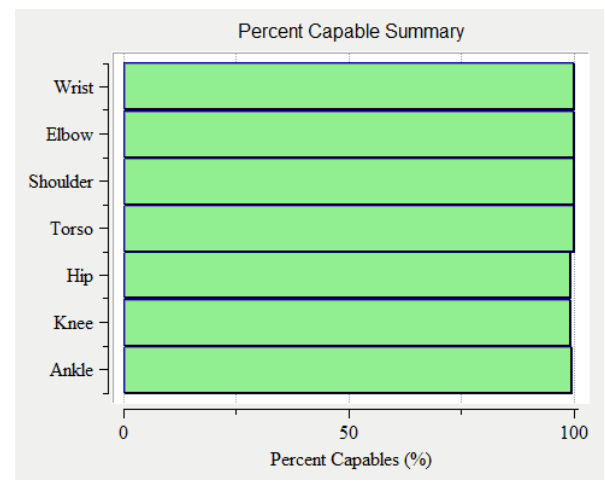

Fig 7. Static strength of driver after optimization

should be slow and excessive, and the pressure between L4 and L5 spines should be reduced as much as possible. As shown in Fig 8, the simulation results show that the driver's seat design reduces the pressure between the driver's L4 and L5, improves the seat comfort, and thus reduces the possibility of lumbar spine diseases.

L4/L5 Forces (N)

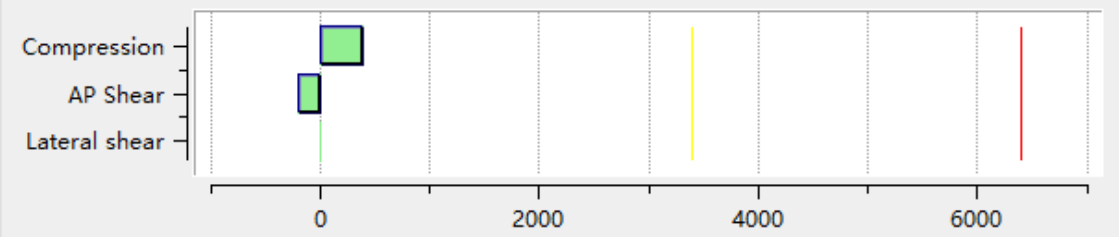

The low back compression force of 390 is below the NIOSH Back Compression Action Limit of $3400 \mathrm{~N}$, representing a nominal risk of low back injury for most healthy workers.

Fig8. Pressure simulation results of L4 and L5 lumbar spine in low back 


\subsubsection{Accessibility Analysis of Agricultural Machinery}

Accessibility analysis of the operator refers to the maximum area that the operator can reach with his hands or feet under a certain posture. The purpose of accessibility analysis is to analyze whether the manual control device or the foot control device is within the

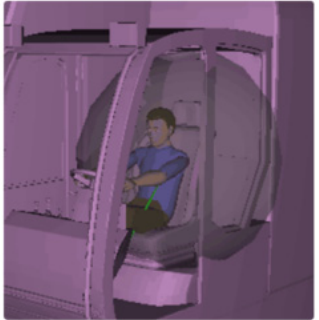

(a)

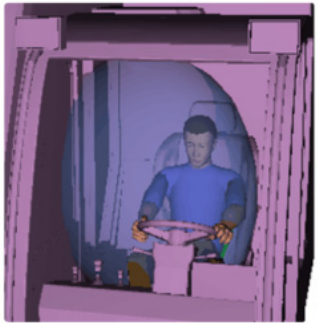

(b)

operation range of the hand or foot, which reflects the rationality of the layout design. The manual control device for agricultural machinery operation includes: touch panel, button, steering wheel, operation rod, etc. Foot control device includes accelerator pedal, brake pedal, etc. Fig 9 shows the reachable simulation system in JACK. The operator has two states during operation.

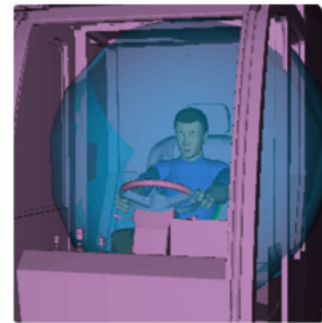

(c)

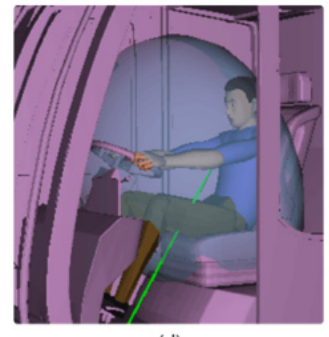

Fig 9. Operator accessibility analysis

The first is the comfortable posture of keeping the back and shoulders motionless with the back seat, and the maximum control area that can be reached only by upper limb movement. Fig 9 (a) shows the left-hand reachable area $L u$, Fig 9 (b) shows the right-hand reachable area $R u$, and the two-hand reachable area is denoted as $U$.

The second is the linkage of the upper limb with the shoulder, back and waist. The maximum control area that can be reached in this state is the left-hand accessible area $L n$ in Fig 9 (c) and the right-hand accessible area $R n$ in Fig 9 (d). The total accessible area in this state is recorded as $N$. Their collective relationship is:

$$
\begin{aligned}
& U=L u+R u \\
& N=L n+R n \\
& U \subseteq N
\end{aligned}
$$

Combined with the importance of the operating device, the operating frequency and safety and the layout principles of human operation accuracy [13-15], the summary is as follows:

$$
\begin{aligned}
& x \in U \\
& y \in N-U
\end{aligned}
$$

Where $\mathrm{x}$ is the set of devices in $U$, with high placement frequency and common control devices, and y is the set of devices in $N$, with low placement frequency and dangerous control devices. Fig 10 is a set relationship of operating devices laid out in two areas.

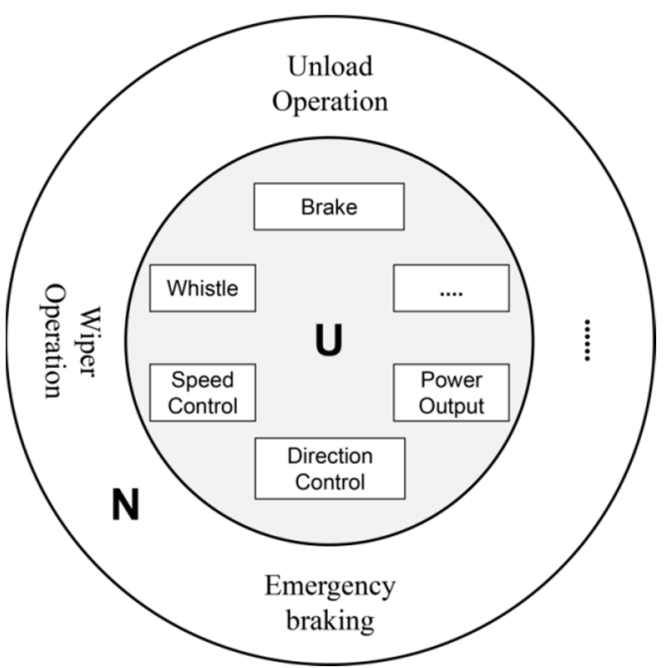

Fig 10. Control device layout and reachable area set diagram

Combined with JACK accessibility analysis and the layout principle of operating devices, the operating devices of Rosa roxburghii picking machine are all in the accessibility area, which shows that the layout is reasonable.

\subsubsection{Accessibility Analysis of Agricultural Machinery}

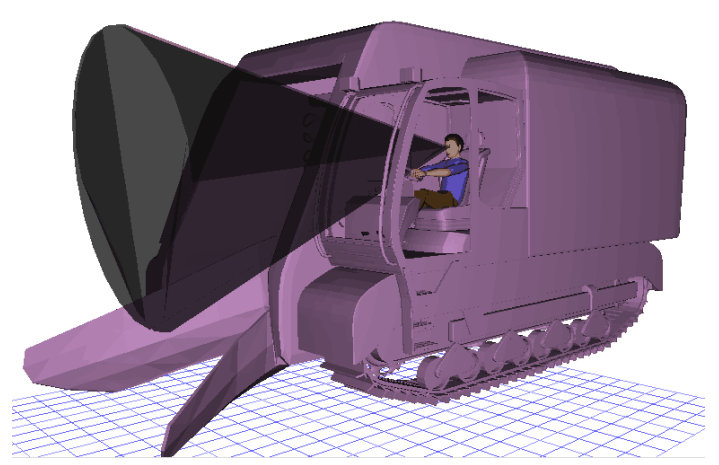

Fig 11. Operator view Cones 
Visibility can directly reflect the operator's visual area and blind spot of operation. In Jack software, View Cones simulation starts from the operator's eyes and sets the cone angle as 40 degrees of human physiological characteristic visual angle. Vision Analysis simulation can intuitively reflect the visual area range of human eye's visual angle and judge the rationality of design through area coverage [3]. As shown in Fig 11, that operator's line of sight can cover the area in front of the agricultural machinery with good visibility.

\section{Conclusion}

In order to improve the coordination of agricultural machinery in the human-machine-environment system, the ergonomic software Jack is used to simulate the operation of Rosa roxburghii picking machine, and the simulation analysis is carried out from the aspects of comfort, accessibility and visibility. Comfort evaluation is carried out on the back, shoulders, hands and other parts of the human body, accessibility is analyzed, and the layout of the corresponding operating devices is deduced based on the principles of importance, operating frequency, safety and accuracy of human body operation of the operating devices, so as to verify the accessibility coverage. Through visibility analysis, the central field of vision of human in the process of mechanical operation is analyzed.

The virtual design simulation research method is more intuitive, improves the rationality of the design, can greatly shorten the research and development cycle, save the design cost, and improve the competitiveness of agricultural machinery products in the market.

\section{Acknowledgments}

Guizhou Science and Technology Plan Project ([2014] 7629, [2017] 7232,[2016] 7432, [2017] 1047); Guizhou Education Department Youth Science and Technology Talents Growth Project, [2018] 112; Guizhou University's Talent Introduction Project, Research on Semantic-Driven Music and Image Emotion Recognition Technology, No.16 [2018].

\section{References}

1. Yan Bai. (2013) Analysis on operation safety of agricultural machinery of china[J]. Journal of Chinese Agricultural Mechanization, 04: 14-17+28.

2. Yulong Wang, Hao Zhong, Jinling Tian, et al. (2017) Development status and trend of agricultural cab technology[J]. Journal of Chinese Agricultural Mechanization, 38(8):130-134.

3. Jianwei Wang, Jianmin Zhang. (2018) Analysis and simulation of ergonomics of agricultural machinery cab based on Jack[J]. Journal of Agricultural Mechanization Research, 40(6):246-251.

4. Fei Yang, Zhenyu Guang, Min Kang. (2017) Ergonomics optimization of tractor cab based on
RULA[J]. Journal of Chinese Agricultural Mechanization, 38(4):88-93.

5. Xiaoping Jin, Yin Chong, Enrong Mao, et al. (2008) Research on Reasoning System for Vehicle ManMachine Interface Layout Optimization[J]. Transactions of the Chinese Society for Agricultural Machinery, 04:183-186.

6. WeiWei Wang, Xin Jin, Suihuai Yu, et al. (2013) The instrument layout design and optimization of rescue vehicles[J]. Machinery Design \& Manfacture, 1001-3997.08.016:51-53.

7. Ligang Wang, Xiugan Yuan, Chunxin Yang. (1997) Study of human model in human-centered manmachine system design[J]. Journal of Beijing University of Aeronautics and Astronautics, (05):5-9.

8. Smith, J., Mansfield, N., Gyi, D., et al.: Driving performance and driver discomfort in an elevated and standard driving position during a driving simulation. Appl. Ergon. 49, 25-33,2015.

9. Xiuquan LI. (2017) Study on the early warning mechanism and equipment design of truck driver fatigue driving[D]. Southwest Jiaotong University.

10. Summerskill, S., Marshall, R., Cook, S., et al. (2016) The use of volumetric projections in digital human modelling software for the identification of large goods vehicle blind spots. Appl.Ergon. 53, 267-280.

11. Gao Fei. (2017) Study of Driver's Fatigue Mechanism and Driving Comfort Evaluation Based on Musculoskeletal Biomechanics [D].Jilin University.

12. Li Wenhao. (2015) Biomechanical response and ergonomics design of the musculoskeletal model based on Anybody[D] Shandong University.

13. Deng Li. (2016) Research on Layout Design and Evaluation Optimization of Human-machine Interface for Cabin[D].Northwestern Polytechnical University.

14. Jianwei Wang, Zhang Jianmin, Yang Qin. (2020) Modeling Design and Evaluation of Rotary Tiller Based on Multidisciplinary Optimization. ICMD 2019, MMS 77, pp. 669-685.

15. Ralph M. (1978) Barnes.Work Methods Manual[M]. John Wiley\&Sons,INC Chapman\&Hall,Ltd. New York.82-84. 